\title{
The role of serum hyaluronic acid determination in the diagnosis of liver fibrosis
}

\author{
Monika Gudowska ${ }^{1 凶}$, Bogdan Cylwik² and Lech Chrostek ${ }^{1}$ \\ 1Department of Biochemical Diagnostics, Medical University of Bialystok, Bialystok, Poland; 2Department of Pediatric Laboratory Diagnostics, \\ Medical University of Bialystok, Bialystok, Poland
}

The common pathway leading to liver fibrosis and cirrhosis is growing deposition of extracellular matrix (ECM). It results from molecular and histological rearrangement of collagens, glycoproteins and hyaluronans. Hyaluronic acid is a chief component of the extracellular matrix of connective tissues and plays the main structural role in the formation of ECM. The most important organ involved in the synthesis of hyaluronic acid is the liver. In this paper the meaning of hyaluronic acid in the diagnostics of liver diseases is discussed. Here, we focus on the described changes of hyaluronic acid concentration in the pathological processes of the liver, including alcoholic and non-alcoholic liver diseases. The results of published clinical studies have shown its high diagnostic sensitivity, which probably enables its application in laboratory diagnosis.

Key words: hyaluronic acid, extracellular matrix, non-invasive marker, cirrhosis, fibrosis, hepatitis

Received: 17 October, 2016; revised: 05 January, 2017; accepted: 15 January, 2017; available on-line: 19 July, 2017

e-mail: monika.gudowska@umb.edu.pl

Abbreviations: HA, hyaluronic acid; ECM, extracellular matrix; HCV hepatitis C virus; HBV, hepatitis B virus; HAV, hepatitis A virus; FL, fatty liver; FLF, fatty liver and mild fibrosis; FLI, fatty liver with moderate fibrosis and inflammation; SFI, severe fibrosis and inflammation; C, cirrhosis; AUCROC, the area under the ROC curve; PIIINP, $\mathrm{N}$-terminal propeptide of procollagen type III; IL-1, interleukin 1; IL-6, interleukin 6; TNF-a, the tumor necrosis factor; NAFLD, nonalcoholic fatty liver disease; NAFL -non-alcoholic fatty liver; $\mathrm{NASH}$ non-alcoholic steatohepatitis; ALD, alcoholic liver diseases; PBC, primary biliary cirrhosis

\section{INTRODUCTION}

Liver fibrosis is characterized by the excessive accumulation of extracellular matrix (ECM) components (Bataller \& Brenner, 2005). It may result from molecular and histological rearrangement of collagens, proteoglycans, glycoproteins and hyaluronans (Gressner et al., 2010). Progressive hepatic fibrosis leads to cirrhosis associated with hepatic dysfunctions and higher risk of morbidity and mortality (Rossi et al., 2007). Cirrhosis typically slowly develops over the years, when the healthy liver tissue is replaced with a fibrous scar (Bataller \& Brenner, 2005). The best known causes of liver fibrosis and cirrhosis are chronic HAV, HBV and HCV infections, alcohol abuse, nonalcoholic steatohepatitis, toxic hepatitis, metabolic conditions such as haemochromatosis and autoimmune hepatitis (Rossi et al., 2007). The symptoms of the liver diseases vary with the stage of the disease. Additionally, in the beginning, liver diseases often progress with non-specific symptoms, shared by a variety of disorders. Currently, diseases of the liver are diagnosed through several methods: physical exams, commercial blood tests such as: bilirubin, albumin, aspartate and alanine aminotransferase and gamma-glutamyltransferase. However, the "gold standard" for the diagnosis and assessment of the liver disease severity is still liver biopsy (Bierman et al., 1957; Gitlin \& Serio, 1992; Bravo et al., 2001). Unfortunately, the liver biopsy has a number of limitations and disadvantages. Some of the drawbacks of biopsy are associated with its invasiveness and with the risk of complications (e.g. bleeding in the liver, pain around the biopsy area). Furthermore, biopsy results are burdened with a large sampling error, as sampled tissue represents only 1/50000 of the whole liver tissue (Rossi et al., 2007; Rostami \& Parsian, 2013). Nowadays, noninvasive diagnostic tests hold a promise for improved diagnosis of the liver diseases. A perfect biomarker should be suitable for the early diagnosis of the disease, useful in determining the prognosis of the disease, safe, quick and easy to test for. Many studies have shown that hyaluronic acid plays an important role in pathogenesis of liver fibrosis and cirrhosis. This review describes and discusses a potential role of hyaluronic acid as a noninvasive biomarker of the liver diseases.

\section{HYALURONIC ACID DEFINITION}

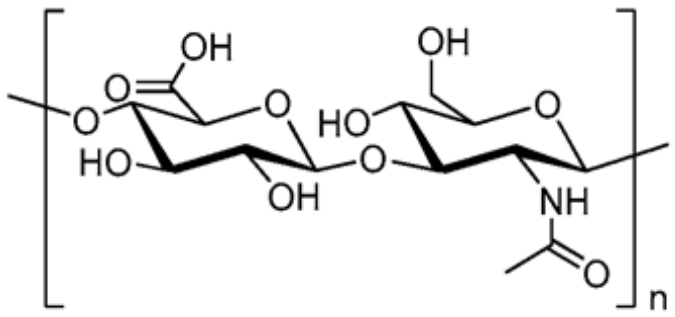

Figure 1. Chemical structure of hyaluronic acid.

Hyaluronic acid (hyaluronan, HA) is a high molecular weight glycosaminoglycan (between 1000 and 10000 $\mathrm{kDa}$ ), which consists of a series of repeated (up to 10000 or more) disaccharides: $\beta-\mathrm{D}-\mathrm{N}$-acetylglucosamine and $\beta$-D-glucuronic acid linked through $\beta-1,4$ and $\beta-1,3$ glycosidic bindings (Fig. 1) (Necas et al., 2008; Kaux et al., 2016). The most important organ involved in the synthesis of hyaluronic acid is the liver. Hyaluronic acid is synthesized in synovial lining cells and hepatic stellate cells (HSC) in a highly controlled process. Enzymes responsible for HA synthesis (the hyaluronic acid synthases) are located at the inner surfaces of the plasma membranes, in particular in the liver cells (Rossi et al., 
2007; Rostami \& Parsian, 2013). Humans have three types of hyaluronan synthases: HAS1, HAS2 and HAS3 which are working by adding activated forms of sugars, UDP-glucuronic acid and UDP-N-acetylglucosamine, to the growing oligosaccharide chain (Necas et al., 2008; Rostami \& Parsian, 2013).

Degradation of hyaluronic acid normally occurs by sinusoidal endothelial cells (Stickel et al., 2003) and in addition by reactive oxygen species (ROS), nitrogen species or by a strictly controlled mechanism involving three types of enzymes: hyaluronidase (Hyal), $\beta$-Dglucuronidase, and $\beta$-N-acetylhexosaminidase (Necas et al., 2008; Schante et al., 2011; Naor, 2016). The most widely expressed hyaluronidases are Hyal-1 and Hyal-2, which cleave high molecular weight hyaluronic acids into smaller oligosaccharides. Degradation of hyaluronic acid in the organism occurs rapidly, thus its half-life in the blood is about 2-5 minutes (Afdhal \& Nunes, 2004; Necas et al., 2008; Schanteet al., 2011). Therefore, an elevated serum hyaluronic acid concentration may be related to impaired clearance and degradation by the liver cells (Stickel et al., 2003).

Hyaluronic acid is practically present in every tissue in the mammalian body, such as joints, muscles, skin, the vitreous body of the eye and liver (Schante et al., 2011; Kaux et al., 2016). This molecule exists in various forms: as a free acid in the lymphatic system and blood stream, or bound to the CD44 receptors on the cell surfaces (Schante et al., 2011; Rostami \& Parsian, 2013). HA is a chief component of the extracellular matrix (ECM) of connective tissues, but it can be also found in the pericellular and intracellular matrix (Necas et al., 2008; Kaux et al., 2016). This polymer plays a main structural role in the formation of ECM due to its viscoelastic properties, hygroscopic capacities and the diversity of cell processes it controls (Nusgens, 2010; Gressner et al., 2010). HA keeps ECM and ECM components (e.g. collagen and elastin fibers) hydrated and stable. It is one of the most hydrophilic molecules in the human body which can bind water, transport and control tissue hydration and maintain an osmotic balance (Necas et al., 2008; Schante et al., 2011; Kaux et al., 2016). There is also evidence that HA plays a role in mitosis, migration, adhesion, tumor development, cancer proliferation and metastasis. Additionally, some authors suggest that high- and low-molecular hyaluronic acid molecules exhibit opposite activity: high-molecular fragments are anti-angiogenic and antiinflammatory, while low-molecular fragments are inflammatory, immune-stimulatory and angiogenic (Rossi et al., 2007; Necas et al., 2008).

\section{ALCOHOLIC LIVER DISEASES (ALD)}

Alcohol is the most commonly abused substance in the world, in the rich and poor countries alike (Ali et al., 2011). Liver is an organ which is especially exposed to ethanol because, after absorption, over $90 \%$ of ethanol is metabolized in the hepatocytes (Bullock, 1990). Alcoholic liver diseases can develop into three forms: fatty liver, alcoholic hepatitis or cirrhosis (Fairbanks, 2012). Chronic alcoholic liver diseases are the major cause of liver transplantations (Naveau et al., 2005). This can be prevented only by detection of fibrosis at an early stage and before the onset of clinical symptoms and liver function decrease (Parkes et al., 2012). One of ways for early detection is the use of non-invasive biomarkers, e.g. hyaluronic acid. Hyaluronic acid serum levels are typically low in healthy individuals, because circulating hyaluronic acid is rapidly removed from the circulation in a receptor-mediated way by the liver endothelial cells. Therefore, liver dysfunction or damage may lead to an increased hyaluronic acid serum concentration. In the study by Stickel and coworkers (2003) a total of 87 subjects underwent ultrasound-guided percutaneous liver biopsy. They were classified into five groups according to histological findings: fatty liver (FL), fatty liver and mild fibrosis (FLF), fatty liver with moderate fibrosis and inflammation (FLI), severe fibrosis and inflammation (SFI) and cirrhosis (C). Hyaluronic acid concentration was measured with a radioimmunoassay kit in the same patients. In this study, the authors had shown that hyaluronic acid concentration was higher in patients when compared to healthy subjects, apart from patients with fatty liver alone, whose level of hyaluronic acid was similar to controls. Moreover, the serum hyaluronan correlated with histological progression of the alcoholic liver disease, reaching the highest levels in patients with alcohol cirrhosis. In addition, hyaluronic acid was elevated in $100 \%$ of patients with cirrhosis and AUCROC (the area under the ROC curve) for predicting the perivenular fibrosis equaled to 0.78 (Stcikel et al., 2003). These results were in accordance with the Naveau and coworkers (2005) study, which revealed that hyaluronic acid levels increased in parallel with the severity of liver damage expressed in the METAVIR scoring system. They have shown that the hyaluronic acid level was higher for stages F4 and F3 in comparison with stages F2, F1 and F0, but there were no significant differences between stages F2 and F1or F0. Similar to results obtained by Stickel and coworkers (2003), the authors had proven that the hyaluronic acid has excellent diagnostic values for the diagnosis of alcoholic cirrhosis with AUC equal to 0.93. These results suggest that hyaluronic acid is a good marker for the detection of advanced liver fibrosis. Furthermore, the authors compared hyaluronic acid detection assay with the FibroTest. They had shown that the FibroTest has some advantages over the hyaluronic acid test. Firstly, it has been standardized and secondly, it has higher sensitivity for the diagnosis of moderate-stage of fibrosis. On the other hand, Parkes and coworkers (2012) had compared the diagnostic power of hyaluronic acid and the N-terminal propeptide of procollagen type III (PIIINP), and the main benefit of hyaluronic acid assessment was an ability to detect inflammation in patients (by observing increased HA concentration), while PIIINP level did not allow distinction between patients with inflammation and controls (Parkes et al. 2012). In our previous study we have shown that the AUC of hyaluronic acid in alcoholic cirrhosis was the highest (0.996), ranking just after the non-patented and noninvasive indicator of liver fibrosis - the GAPRI index (Gudowska et al., 2015). Moreover, $\mathrm{HA}$, as a single marker, has a higher AUC than other complex markers: the Forn's index, APRI, and the FIB4 score. We also calculated the HAPRI index, which is a strong predictor of alcoholic cirrhosis, similar to the hyaluronic acid alone. Additionally, a correlation study demonstrated an association between severity of the liver cirrhosis (evaluated by the Child-Pugh scale) and the hyaluronic acid serum concentration. According to these studies, the hyaluronic acid concentrations were the highest at the most severe stage of the liver injury (Child-Pugh class C) (Gudowska et al., 2015). Most of the publications suggest that high hyaluronic acid concentration is related to a decreased function and "capillarization" of endothelial sinusoidal cells responsible for degradation of excess ECM (Deaciuc IV et al., 1993; Deaciuc IV et al., 1994; Stickelet al., 2011; Parkes et al., 
2012). The "capillarization of the sinusoids" efficiently prevents elimination of hyaluronic acid form circulation (Stickel et al., 2003). Furthermore, studies performed on mice and rat models have shown that alcohol affects this glycosaminoglycan level by modifications of hepatic hyaluronic acid clearance (Deaciuc IV et al., 1994). Chronic alcohol consumption may also lead to an increase in interleukin 1 (IL-1), interleukin 6 (IL-6) and the tumor necrosis factor (TNF- $\alpha$ ). These inflammatory cytokines may stimulate hepatic stellate cells to produce hyaluronic acid (Stickel et al., 2003).

On the basis of our own experience and literature data we suggest that hyaluronic acid is a good marker for diagnosis and staging of the alcoholic liver diseases. In addition, hyaluronic acid has an excellent diagnostic value for the diagnosis of advanced fibrosis and alcoholic liver cirrhosis. Therefore, as clinical biochemistry quickly develops, and the understanding of hyaluronic acid mechanism of action and clinical potential deepens, the eventual use of hyaluronic acid in clinical practice seems to be assured.

\section{NON-ALCOHOLIC FATTY LIVER DISEASE (NAFLD)}

Nonalcoholic fatty liver disease, characterized by accumulation of lipids in hepatocytes, is becoming a major liver disease in the developing Western countries. NAFLD can be divided into non-alcoholic fatty liver (NAFL) and non-alcoholic steatohepatitis (NASH) (Kneeman et al., 2012; Chalasani et al., 2012). A metabolic syndrome and liver transplantation remain the main causes of these diseases. Both of these conditions can progress to a liver fibrosis and cirrhosis (Gitto \& Villa, 2016). Therefore, an early detection of NAFLD and distinguishing non-alcoholic and alcoholic chronic liver diseases is very important. To this end Sowa et al had compared non-invasive markers, including hyaluronic acid, in patients with NAFLD and alcoholic liver diseases (Sowa et al., 2013). They had found that adiponectin and tumor necrosis factor- $\alpha$ were significantly lower, and the ratio of alanine aminotransferase to aspartate aminotransferase was higher, in patients with non-alcoholic fatty liver disease when compared to alcoholic patients with and without liver cirrhosis. Hyaluronic acid concentrations were similar in the liver diseases caused by alcohol and non-alcoholic factors (Sowa et al., 2013). In contrast, hyaluronic acid levels helped to distinguished the non-alcoholic fatty liver from non-alcoholic steatohepatitis. Although the patients with NASH had a significantly increased expression of HA, patients with stage 1 of NASH expressed a similar concentration of HA to patients with NAFL. This may suggest that hyaluronic acid is a worse discriminator of NASH and NAFL at an early stage of fibrosis than other markers, e.g. type IV collagen and collagen 7S (Mizuno et al., 2016). Moreover, Chwist and coworkers (2014) reported that the serum level of hyaluronic acid correlates well with the progression (from F0 to F3) and extension of liver fibrosis in patients with NAFLD (Chwist et al., 2014). In addition, Dvorak and coworkers (2014) had shown that serum concentrations of hyaluronic acid were higher in patients with advanced fibrosis than in those suffering from mild fibrosis. At a cut-off point of $>25 \mu \mathrm{g} / \mathrm{L}$, hyaluronic acid discriminates patients with stages $\mathrm{F} 3-\mathrm{F} 4$ from those with no or mild fibrosis (F0-F2), with high sensitivity and specificity ( $90 \%$ and $84 \%$, respectively). Hyaluronic acid as a marker of fibrosis was also measured in children with NAFLD (Fitzpatrick et al., 2010; Lebensztejn et al.,
2011). Fitzpatrick and coworkers (2010) revealed that its concentration was significantly higher in the NAFLD patients with fibrosis than in controls. Similar results were obtained by Lebensztejn and coworkers (2011) who studied four potential serum markers of liver fibrosis. They discovered that hyaluronic acid and cytokeratin-18 M30 are elevated in children with NAFLD. They had also shown the ability to differentiate NAFLD patients with fibrosis from those without fibrosis (AUC=0.672) at a hyaluronic acid cut-off point of $19.10 \mathrm{ng} / \mathrm{mL}$, and an improved ability of differentiation for the combination of HA with cytokeratin-18 M30 (AUC=0.730). Moreover, Stachowska and coworkers (2013) suggested that a significant difference in hyaluronic acid concentration, as well as predicted advanced fibrosis, occurs in patients with Apo-E4 alleles. Nonetheless, Nguyen-Khacet and coworkers (2008) had compared the performance of Fibroscan with seven non-invasive laboratory tests, including hyaluronic acid and Hepascore, which comprised of $\alpha-2$ macroglobulin, hyaluronic acid, GGT, and total bilirubin adjusted by age and gender. They revealed that the performance of Fibroscan was higher than that of laboratory tests, for which AUROCs ranged from 0.66 to 0.77 (F1), 0.54-0.82 (F2), 0.43-0.88 (F3) and 0.560.89 (F4). In addition, combining Fibroscan with each test did not improve its diagnostic utility which can suggest that noninvasive transient elastography used to assess liver stiffness is a better alternative for liver biopsy than serum biomarkers.

These studies indicate that hyaluronic acid level determination can help to quickly evaluate the progression of non-alcoholic liver diseases. Hyaluronic acid is a good marker to differentiate NAFLD patients with fibrosis form those without fibrosis, and to distinguish nonalcoholic fatty liver from non-alcoholic steatohepatitis. However, it's not an adequate indicator of alcoholic vs. non-alcoholic liver disease.

\section{CHRONIC HEPATITIS C}

Up to $80 \%$ of hepatitis patients develop chronic hepatitis $\mathrm{C}(\mathrm{CHC})$. Chronic hepatitis $\mathrm{C}$ infection induces continuous inflammation in the liver, progression of hepatic fibrosis, eventual cirrhosis, and possible hepatocellular carcinoma (Nelson et al., 2011). Most of the studies had shown elevated hyaluronic acid serum levels in chronic hepatitis C. Additionally, few studies found that hyaluronic acid may differentiate the stages of liver damage. HA might be associated with long-lasting chronic liver injury and fibrosis, growing production of extracellular matrix components and dwindling hepatic clearance (Korner et al., 1996; Halfon et al., 2005; Rossi et al., 2007).

Mehta and coworkers (2008) had found that serum hyaluronic acid levels correlated with digital quantification of fibrosis (DQF) and Ishak stages in chronic hepatitis C. They had shown that hyaluronic acid could help to discriminate between intermediate fibrosis (Ishak stages 2-3) and absent/minimal fibrosis (Ishak stages $0-1$ ). Hyaluronic acid assessment has also proven to have better diagnostic power than FIBROSpect II (a diagnostic panel of three extracellular matrix remodeling markers: a2-macroglobulin, tissue inhibitor of metalloproteinases- 1 and hyaluronic acid). In addition, the proportion of false-positive results in case of hyaluronic acid (33\%) was lower in comparison with FIBROSpect II and YKL40 (chondrex, human cartilage glycoprotein-39) (Mehta et al., 2008). In turn, Fontana and coworkers (2008) revealed that a 3-variable model (hyaluronic acid, tissue in- 
hibitor of metalloproteinases- 1 and platelet count) is an excellent indicator for estimating the presence of cirrhosis (Ishak stages $>5$ ) in patients with chronic hepatitis $C$ (AUROC - 0.81) participating in "The hepatitis $C$ antiviral long-term treatment against cirrbosis" (HALT-C Trial) (Fontana et al., 2008). They had also shown that this model is significantly better in identifying hepatitis C patients than other published models, but they agreed that the serum fibrosis markers reflect the stage of fibrosis more precisely than the quantity of hepatic collagen.

The significant difference in hyaluronic acid level among fibrosis stages (5-point METAVIR scale) in HCV patients was also estimated by Arain and coworkers (2011), but the negative predictive value was low for a significant liver disease (Arain et al., 2011). Moreover, positive predictive value at the level of $60 \mathrm{ng} / \mathrm{mL}$ (only in $15 \%$ of the patients) equaled to $85 \%$. Conversely, McHutchison and coworkers (2000) had shown that the hyaluronic acid value $<60 \mathrm{ng} / \mathrm{mL}$ excluded the presence of cirrhosis or advanced fibrosis with a $99 \%$ and 93\% positive predictive value, respectively. These authors demonstrated that the concentration of HA determined with a radioimmunoassay is significantly lower in the non-cirrhotic patients and patients with no fibrosis when compared to patients with cirrhosis and fibrosis, respectively. In addition, our previous study had shown that in $39 \%$ of cases of non-alcoholic cirrhosis caused by chronic hepatitis C (at the cut-off point of $72 \mathrm{ng} / \mathrm{mL}$ ), the hyaluronic acid has a very good diagnostic power (positive predictive value $-100 \%$, sensitivity - $77.3 \%$ and AUC - 0.884). When hyaluronic acid was combined in the HAPRI algorithm with indirect marker of hepatic fibrosis - INR - the AUROC (0.970) was slightly higher than for HA alone. This suggests that non-invasive algorithms are more useful than a single marker, such as hyaluronic acid level (Gudowska et al., 2015). Valva and coworkers (2011) seems to agree with the fact that combination of a few markers (HA, PIIINP and TGF- $\beta$ ) has greater diagnostic accuracy in recognition of fibrosis compared to a single marker. Furthermore, El-Bassiouni and coworkers (2012) had examined 120 patients with chronic hepatitis C, from which 30 had liver cirrhosis and 30 had hepatocellular carcinoma. They determined the serum HA, platelet-derived growth factor, expression of connective tissue growth factor and transforming growth factor, and observed a significant increase in hyaluronic acid concentration and connective tissue growth factor expression in all patients. These data revealed that both, the hyaluronic acid and connective tissue growth factor may be used as important diagnostic parameters for assessment of hepatic fibrosis.

Therefore, hyaluronic acid has a potential to be used for the detection of chronic hepatitis C, evaluation of fibrosis degree and detection/differentiation of intermediate stages of fibrosis, starting from minimal fibrosis. In addition, it is a good tool to exclude cirrhosis or advanced fibrosis in chronic hepatitis C patients (when hyaluronic acid value is lower than $60 \mathrm{ng} / \mathrm{mL}$ ).

\section{CHRONIC HEPATITIS B}

Chronic hepatitis B virus (HBV) infection is a major world health problem because about a third of world's population has serological evidence of past or present infections with $\mathrm{HBV}$, including 350 million people with chronic infections. The early detection of HBV-induced liver injury is an important objective, because in some cases HBV-infections progress to cirrhosis and liver fail- ure or liver cancer (Schilsky, 2013; Hsu et al., 2002). It has been suggested that hyaluronic acid may be a good biomarker of HBV infection. Study of Geramizadeh and coworkers (2008) had shown that the serum hyaluronic acid levels correlate with the degree of fibrosis according to the criteria proposed by the Ishak system. Hyaluronic acid reaches the highest concentration in the group with extensive liver fibrosis. In this group of patients, the hyaluronic acid level was about 2 -times higher in comparison with stages 3-4 of the Ishak scoring system, and almost 5-times higher than that in stages $0-2$. The much higher concentration of hyaluronan in the group with extensive fibrosis or cirrhosis suggests that hyaluronic acid is a precise predictor of the terminal stages of liver damage in patients with HBV (Geramizadeh et al., 2008). Besides the fact that hyaluronic acid has the best diagnostic accuracy (AUROC 0.902) for predicting fibrosis of stages 3 or more, Gumusay and coworkers (2013) had demonstrated a better diagnostic value of an Enhanced Liver Fibrosis test (ELF) in predicting advanced fibrosis. ELF is combination of hyaluronic acid, procollagen III amino terminal peptide and tissue inhibitor of metalloproteinase 1 and APRI index (combination of platelet count and aspartate aminotransferase). In a study that monitored liver fibrosis in children during IFN $\alpha$ treatment, hyaluronic acid concentration was significantly lower after 12 months of INF- $\alpha$ treatment in comparison with the level before treatment (Lebensztejn et al., 2006). This suggests that hyaluronic acid may be an important factor allowing to discriminate between patients with liver fibrosis and healthy individuals, and monitoring the treatment of chronic hepatitis B. Additionally, in the study of $\mathrm{Li}$ and coworkers (2012) the diagnostic performance of serum hyaluronic acid for predicting significant fibrosis was found to be better than that of laminin. Hyaluronic acid has higher sensitivity $(84.2 \%)$, specificity $(83.3 \%)$, positive predictive value $(90.6 \%)$ and negative predictive value $(73.5 \%)$ when compared to laminin $(71.9 \%$, $80.0 \%, 87.2 \%$ and $60.0 \%$, respectively). On the other hand, when hyaluronic acid was combined with laminin, it increased the positive predictive value $(100.0 \%)$ and specificity $(100.0 \%)$, but sensitivity had declined $(63.2 \%)$. A study that compared eight biomarkers (procollagen III amino terminal peptide and tissue inhibitor of metalloproteinase 1 , tenascin- $\mathrm{C}$, laminin, matrix metallopeptidase 9, collagen type IV and VI, hyaluronic acid) proved that hyaluronic acid, tissue inhibitor of metalloproteinase 1 and their combination are the most powerful among the fibrosis markers (Seven et al., (2011).

In summary, hyaluronic acid determination can be used to detect chronic hepatitis $\mathrm{B}$ in patients and can illustrate the degree of liver fibrosis. Hyaluronic acid has a very good diagnostic accuracy for predicting advanced fibrosis and may be an important factor to monitor the treatment of chronic hepatitis B.

\section{HIV/HCV COINFECTION}

HIV co-infection accelerates progression of liver fibrosis in the HCV-infected patients. Alarmingly, the highest number of liver-related deaths has been reported in HIV-infected patients co-infected with the hepatitis $\mathrm{C}$ virus. The precise assessment of the degree of liver fibrosis is necessary to begin patients' treatment and therefore it is important to find a perfect marker of liver damage (Benhamou et al., 1999; Salmon-Ceron et al., 2005). Peters and coworkers (2013) had investigated serum hyaluronic acid levels in patients with HIV/HCV 
co-infection and showed that hyaluronic acid concentration was about 7-times higher in patients with liverrelated death or liver coma (LRE) when compared to those without. Moreover, the level of hyaluronan had increased substantially prior to developing LRE and was significantly higher than that in healthy people. Peters and coworkers (2008) had presented a view that the increasing level of this polysaccharide increases the risk of hepatic complications. Resino and coworkers (2010) had compared hyaluronic acid diagnostic performance with different non-invasive algorithms: HGM-1 (based on platelet count, aspartate aminotransferase, glucose), HGM-2 (platelet count, international normalized ratio, alkaline phosphatase, aspartate aminotransferase), Forns (gamma-glutamyltransferase, age, platelet count, cholesterol), APRI (platelet count, aspartate aminotransferase) and FIB-4 (age, aspartate aminotransferase, alanine aminotransferase, platelet count). The results obtained by these authors revealed that simple hyaluronic acid assessment is a better marker than complex indexes. The AUC increased with the stage of fibrosis, reaching the highest value $(0.863)$ in the last stages of fibrosis. The values of AUC were decreased in mild or moderate fibrosis and advanced fibrosis (0.676 and 0.772, respectively). Moreover, Nunes and coworkers (2005) had compared the diagnostic performance and characteristics of noninvasive markers of hepatic fibrosis including hyaluronic acid in HCV patients with and without HIV infection. They had shown that correlations between hyaluronic acid and other fibrosis markers with the stage of fibrosis were similar in the groups with and without HIV infection.

Taking into account the current literature data, hyaluronic acid has better diagnostic performance than complex algorithms and an increasing level of this polysaccharide in HIV/HCV co-infected patients is associated with the risk of hepatic complications.

\section{OTHER DISEASES}

$\mathrm{Yu}$ and coworkers (2015) had found a significant increase of hyaluronic acid, laminin, type III serum procollagen peptide and type IV collagen concentrations in elderly patients during schistosome-induced liver disease complicated by hepatitis E, compared to senile patients with simple hepatitis E. Mima and coworkers (2014) suggested that hyaluronic acid probably plays a critical role in tumorigenesis. These authors had analyzed the relationship between preoperative hyaluronic acid serum concentration and prognosis after hepatic resection in patients with hepatocellular carcinoma and revealed that high serum hyaluronic acid levels $(>100 \mathrm{ng} / \mathrm{mL})$ predict poor prognosis in patients with hepatocellular carcinoma after hepatic resection. Moreover, Shen and coworkers (2015) used data from 89 patients with hepatocellular carcinoma to create an algorithm including 4 parameters: preoperative HBV DNA level, serum prealbumin, laminin and hyaluronic acid. Their method reached the sensitivity of $62.5 \%$ and specificity of $91.2 \%$. However, there was no correlation between postoperative liver function and the overall survival. These results may suggest that hyaluronic acid is a better predictor of postoperative liver dysfunction than a combination of a few other markers.

Voumvouraki and coworkers (2011) suggest that serum hyaluronan is a promising single serum marker for longitudinal studies in primary biliary cirrhosis (PBC). They have determined four markers: hyaluronic acid, leptin, laminin and collagen IV in the peripheral and hepatic vein blood of PBC patients. Sera from the PBC group were compared to the controls, patients with hepatitis C, hepatocellular carcinoma and viral cirrhosis. According to this study hyaluronic acid and collagen IV were increased in all liver diseases when compared to

Table 1. Summary of literature review about hyaluronic acid importance for detection, differentiation and staging of liver diseases.

\begin{tabular}{|c|c|}
\hline Importance for: & Type of Liver Disease \\
\hline \multicolumn{2}{|c|}{ Alcoholic Liver Diseases } \\
\hline Detection & Stickel et al. (2003), Parkes et al. (2012), Gudowska et al. (2015) \\
\hline Differentiation & Sowa et al. (2013), Gudowska et al. (2015) \\
\hline Staging & Stickel et al. (2003), Naveau et al., Gudowska et al. (2015) \\
\hline \multicolumn{2}{|c|}{ Non-Alcoholic Liver Diseases } \\
\hline Detection & Mizuno et al. (2016), Fitzpatrick et al. (2010) \\
\hline Differentiation & Sowa et al. (2013), Gudowska et al. (2015), Mizuno et al. (2016), Chwist et al. (2014) \\
\hline Staging & Sowa et al. (2013), Chwist et al. (2014), Nguyen-Khac et al. (2008) \\
\hline \multicolumn{2}{|c|}{ Chronic Hepatitis C } \\
\hline Detection & $\begin{array}{l}\text { Rossi et al. (2007), Halfon et al. (2005), Korner et al. (1996), Mehta et al. (2008), Fontana et al. (2008), McHutchi- } \\
\text { son et al. (2000), Valva et al. (2011), El-Bassiouniet et al. (2012) }\end{array}$ \\
\hline Differentiation & - \\
\hline Staging & $\begin{array}{l}\text { Rossi et al. (2007); Halfon et al. (2005), Korner et al. (1996), Mehta et al. (2008), Fontana et al. (2008), Arain et } \\
\text { al. (2011), McHutchison et al. (2000) }\end{array}$ \\
\hline \multicolumn{2}{|c|}{ Chronic Hepatitis B } \\
\hline Detection & Geramizadeh et al. (2008), Gumsay et al. (2013), Rostami et al. (2013) \\
\hline Differentiation & - \\
\hline Staging & Geramizadeh et al. (2008), Rostami et al. (2013) \\
\hline \multicolumn{2}{|c|}{ HIV-HCV Conifection } \\
\hline Detection & Peters et al. (2013) \\
\hline Differentiation & Peters et al. (2013), Nunes et al. (2005) \\
\hline Staging & Resino et al. (2010), Nunes et al. (2005) \\
\hline
\end{tabular}


controls, but only hyaluronic acid levels were statistically different between the early and late stages of primary biliary cirrhosis. In addition, hyaluronic acid has the highest AUROC (0.720) for the identification of the late PBC.

Crawford and coworkers (2009) had examined patients with $\mathrm{C} 282 \mathrm{Y}$ hereditary hemochromatosis and suggested that patients with serum ferritin $>1000 \mu \mathrm{g} / \mathrm{L}$ were at a risk of cirrhosis, but only $40 \%$ of patients with serum ferritin $>1000 \mu \mathrm{g} / \mathrm{L}$ were cirrhotic. Therefore, they tried to evaluate the diagnostic utility of other non-invasive fibrosis markers such as hyaluronic acid. They had shown that serum hyaluronic acid was increased in hereditary hemochromatosis patients when compared with the control group. An elevated hyaluronic acid concentration (>46.5 ng/mL) was 100\% sensitive and 100\% specific in identifying patients with liver cirrhosis. Moreover, among patients with high serum ferritin $(>1000 \mu \mathrm{g} / \mathrm{L})$, hyaluronic acid levels were significantly increased in patients with liver cirrhosis in comparison to those without cirrhosis.

Summary of literature review about hyaluronic acid importance for detection, differentiation and staging of liver diseases is presented in Table 1.

\section{CONCLUSION}

Hyaluronic acid serum concentrations are elevated in liver diseases associated with fibrosis, but vary in liver diseases of different etiologies. Additionally, hyaluronic acid levels rise continuously with the severity of liver fibrosis. The significant increase of hyaluronic acid levels in the sera creates the possibility of applying its measurement in the diagnostics of liver diseases. It may be an additional clinical tool for the evaluation of severity of liver diseases when the liver biopsy is impossible to perform. The measurements of hyaluronic acid concentrations could be an excellent indicator of liver fibrosis, because it is an easy to use, simple, quick and non-invasive test.

\section{REFERENCES}

Afdhal NH, Nunes D (2004) Evaluation of liver fibrosis: a concise review. Am J Gastroenterol 99: 1160-1174. doi: 10.1111/j.15720241.2004.30110.x

Ali SF, Onaivi ES, Dodd PR, Cadet JL, Schenk S, Kuhar MJ, Koob GF (2011) Understanding the global problem of drug addiction is a challenge for IDARS cientists. Curr Neuropharmacol 9: 2-7. doi: 10.2174/157015911795017245

Arain SA, Meo SA, Jamal Q (2011) Serum hyaluronic acid level does not reliably differentiate minimal and significant liver disease in chronic hepatitis C. Saudi Med J 32: 1241-1245

Bataller, Brenner DA (2005) Liverfibrosis. J Clin Invest 115: 209-218. doi: $10.1172 / \mathrm{JCI} 24282$

Benhamou Y, Bochet M, Di Martino V, Charlotte F, Azria F, Coutellier A, Vidaud M, Bricaire F, Opolon P, Katlama C, Poynard T (1999)Liver fibrosis progression in human immunodeficiency virus and hepatitis $\mathrm{C}$ virus coinfected patients. The Multivirc Group. Hepatol Baltim Md 30: 1054-1058. doi: 10.1002/hep.510300409

Bierman HR, Hill BR, Reinhardt L, Emory E (1957). Correlation of serum lactic dehydrogenase activity with the clinical status of patients with cancer, lymphomas, and leukemias. Cancer Res 17: 660-667

Bravo AA, Sheth SG, Chopra S (2001) Liver biopsy. N Engl J Med 344: 495-500. doi: 10.1056/NEJM200102153440706

Bullock C (1990) The biochemistry of alcohol metabolism - a brief review. Biochemical Education 18: 62-66

Chalasani N, Younossi Z, Lavine JE, Diehl AM, Brunt EM, Cusi K, Charlton M, Sanyal AJ, American Gastroenterological Association; American Association for the Study of Liver Diseases; American College of Gastroenterologyh (2012) The diagnosis and management of non-alcoholic fatty liver disease: practice Guideline by the American Association for the Study of Liver Diseases, American College of Gastroenterology, and the American Gastroenterological Association. Hepatology 55: 2005-2023. doi: 10.1002/hep.25762
Chwist A, Hartleb M, Lekstan A, Kukla M, Gutkowski K, Kajor M (2014) A composite model including visfatin, tissue polypeptidespecific antigen, hyaluronic acid, and hematological variables for the diagnosis of moderate-to-severe fibrosis in nonalcoholic fatty liver disease: a preliminary study. Pol Arch Med Wewn 124: 704-712 (in Polish)

Crawford DH, Murphy TL, Ramm LE, Fletcher LM, Clouston AD, Anderson GJ, Subramaniam VN, Powell LW, Ramm GA (2009) Serum hyaluronic acid with serum ferritin accurately predicts cirrhosis and reduces the need for liver biopsy in C282Y hemochromatosis. Hepatology 49: 418-425. doi: 10.1002/hep.22650

Deaciuc IV, McDonough KH, Bagby GJ, Spitzer JJ (1993) Alcohol consumption in rats potentiates the deleterious effect of Gramnegative sepsis on hepatic hyaluronan uptake. Alcohol Clin Exp Res 17: 1002-1008

Deaciuc IV, Spitzer JJ, Shellito JE, D’Souza NB (1994) Acute alcohol administration to mice induces hepatic sinusoidal endothelial cell dysfunction. Int Hepat Commun 2: 81-86

Dvorak K, Stritesky J, Petrtyl J, Vitek L, Sroubkova R, Lenicek M, Smid V, Haluzik M, Bruha R (2014) Use of non-invasive parameters of non-alcoholic steatohepatitis and liver fibrosis in daily practice - an exploratory case-control study. PLoS One 9: e111551. doi: 10.1371/journal.pone.0111551

El-Bassiouni NE, Nosseir MM, Madkour ME, Zoheiry MM, Bekheit IW, Ibrahim RA, Ibrahim IM, El Bassiouny AE (2012) Role of fibrogenic markers in chronic hepatitis $\mathrm{C}$ and associated hepatocellular carcinoma. Mol Biol Rep 39: 6843-6850. doi: 10.1007/s11033$012-1509-z$

Fairbanks KD (2012) Alcoholic liver disease http://www.clevelandclinicmeded.com/medicalpubs/diseasemanagement/hepatology/alcoholic-liver-disease/. November 2012

Fitzpatrick E, Mitry RR, Quaglia A, Hussain MJ, DeBruyne R, Dhawan A (2010) Serum levels of CK18 M30 and leptin are useful predictors of steatohepatitis and fibrosis in paediatric NAFLD. J Pediatr Gastroenterol Nutr 51: 500-506. doi: 10.1097/MPG.0b013e3181e376be

Fontana RJ, Goodman ZD, Dienstag JL, Bonkovsky HL, Naishadham D, Sterling RK, Su GL, Ghosh M, Wright EC, HALT-C Trial Group (2008) Relationship of serum fibrosis markers with liver fibrosis stage and collagen content in patients with advanced chronic hepatitis C. Hepatology 47: 789-798. doi: 10.1002/hep.22099

Geramizadeh B, Janfeshan K, Saberfiroozi M (2008) Serum hyaluronic acid as a noninvasive marker of hepatic fibrosis in chronic hepatitis B. Saudi J Gastroenterol 14: 174-177. doi: 10.4103/1319-3767.43274

Gitlin N, Serio KM (1992) Ischemic hepatitis: widening horizons. Am J Gastroenterol 87: 831-836

Gitto S, Villa E (2016) Non-alcoholic fatty liver disease and metabolic syndrome after liver transplant. Int J Mol Sci 17: 490. doi: 10.3390/ ijms 17040490

Gressner AM, Rizk M, Chunfang G, Olav AG (2010) Potential novel biomarkers for monitoring the fibrogenic process in liver. Arab J Gastroenterol 10: S12-S16

Gudowska M, Gruszewska E, Panasiuk A, Cylwik B, Flisiak R, Świderska M, Szmitkowski M, Chrostek L (2015) Hyaluronic acid concentration in liver diseases. Clin Exp Med. doi: 10.1007/s10238015-0388-8

Gümüşay O, Ozenirler S, Atak A, Sönmez C, Ozkan S, Tuncel AF, Yilmaz G, Akyol G (2013) Diagnostic potential of serum direct markers and non-invasive fibrosis models in patients with chronic hepatitis B. Hepatol Res 43: 228-237. doi: 10.1111/j.1872034X.2012.01057.x

Halfon P, Bourlière M, Pénaranda G, Deydier R, Renou C, Botta-Fridlund D, Tran A, Portal I, Allemand I, Rosenthal-Allieri A, Ouzan D (2005) Accuracy of hyaluronic acid level for predicting liver fibrosis stages in patients with hepatitis $C$ virus. Comp Hepatol 4: 6. doi: 10.1186/1476-5926-4-6

Hsu YS, Chien RN, Yeh CT, Sheen I-S, Chiou H-Y, Chu C-M, Liaw Y-F (2002) Long-term outcome after spontaneous HBeAg seroconversion in patients with chronic hepatitis B. Hepatology 35: 15221527. doi: 10.1053/jhep.2002.33638

Kaux JF, Samson A, Crielaard JM (2016) Hyaluronic acid and tendon lesions. Muscles Ligaments Tendons J 5: 264-269. doi: 10.11138/ $\mathrm{mltj} / 2015.5 .4 .264$

Kneeman JM, Misdraji J, Corey KE (2012) Secondary causes of nonalcoholic fatty liver disease. Therap Adv Gastroenterol 5: 199-207. doi: 10.1177/1756283X11430859

Korner T, Kropf J, GressnerAM (1996) Serum laminin and hyaluronan in liver cirrhosis markers of progression with high prognostic value. J Hepatol 25: 684-688

Lebensztejn DM, Sobaniec-Lotowska ME, Kaczmarski M, Voelker M, Schuppan D (2006) Matrix-derived serum markers in monitoring liver fibrosis in children with chronic hepatitis B treated with interferon alpha. World J Gastroenterol 12: 3338-3343

Lebensztejn DM, Wierzbicka A, Socha P, Pronicki M, Skiba E, Werpachowska I, Kaczmarski M (2011) Cytokeratin-18 and hyaluronic acid levels predict liver fibrosis in children with non-alcoholic fatty liver disease. Acta Biochim Pol 58: 563-566 
Li F, Zhu CL, Zhang H, Huang H, Wei Q, Zhu X, Cheng XY (2012) Role of hyaluronic acid and laminin as serum markers for predicting significant fibrosis in patients with chronic hepatitis B. Braz J Infect Dis 16: 9-14.

McHutchison JG, Blatt LM, de Medina M, Craig JR, Conrad A, Schiff ER, Tong MJ (2000) Measurement of serum hyaluronic acid in patients with chronic hepatitis C and its relationship to liver histology. Consensus Interferon Study Group. J Gastroenterol Hepatol 15 . 945-951

Mehta P, Ploutz-Snyder R, Nandi J, Rawlins SR, Sanderson SO, Levine RA (2008) Diagnostic accuracy of serum hyaluronic acid, FIBROSpect II, and YKL-40 for discriminating fibrosis stages in chronic hepatitis C. Am J Gastroenterol 103: 928-936. doi: 10.1111/j.15720241.2007.01761.x

Mima K, Beppu T, Ishiko T, Chikamoto A, Nakagawa S, Hayashi H, Watanabe M, Sakamaki K, Baba H (2014) Preoperative serum hyaluronic acid level as a prognostic factor in patients undergoing hepatic resection for hepatocellular carcinoma. Br J Surg 101: 269-276. doi: $10.1002 /$ bjs. 9343

Mizuno M, Shima T, Oya H, Mitsumoto Y, Mizuno C, Isoda S, Kuramoto M, Taniguchi M, Noda M, Sakai K, Koyama N, Okanoue T (2016) Classification of patients with nonalcoholic fatty liver disease using rapid immunoassay of serum type IV collagen compared with that using liver histology and other fibrosis markers. Hepatol Res doi: 10.1111/hepr.12710 [Epub ahead of print]

Naor D (2016) Editorial: Interaction Between Hyaluronic Acid and Its Receptors (CD44, RHAMM) Regulates the activity of inflammation and cancer. Front Immunol 7: 39. doi: 10.3389/fimmu.2016.00039

Naveau S, Raynard B, Ratziu V, Abella A, Imbert-Bismut F, Messous D, Beuzen F, Capron F, Thabut D, Munteanu M, Chaput JC, Poynard T (2005) Biomarkers for the prediction of liver fibrosis in patients with chronic alcoholic liver disease. Clin Gastroenterol Hepatol 3: $167-174$

Necas J, Bartosikova L, Brauner P, Kolar J (2008) Hyaluronic acid (hyaluronan): a review. Veterinari Medicina 8: 397-411

Nelson PK, Mathers BM, Cowie B, Hagan H, Des Jarlais D, Horyniak D, Degenhardt L (2011) Global epidemiology of hepatitis B and hepatitis $\mathrm{C}$ in people who inject drugs: results of systematic reviews. Lancet 378: 571-583. doi: 10.1016/S0140-6736(11)61097-0

Nguyen-Khac E, Chatelain D, Decrombecque C, Robert B, Joly JP, Brevet M, Grignon P, Lion S, Le Page L, Dupas JL (2008) Assessment of asymptomatic liver fibrosis in alcoholic patients using fibroscan: prospective comparison with seven non-invasive laboratory tests. Aliment Pharmacol Ther 28: 1188-1198. doi: 10.1111/j.13652036.2008.03831.x

Nunes D, Fleming C, Offner G, O’Brien M, Tumilty S, Fix O, Heeren T, Koziel M, Graham C, Craven DE, Stuver S, Horsburgh CR Jr (2005) HIV infection does not affect the performance of noninvasive markers of fibrosis for the diagnosis of hepatitis $C$ virus-related liver disease. I Acquir Immune Defic Syndr 40: 538-544

Nusgens BV (2010) Hyaluronic acid and extracellular matrix: a primitive molecule? Ann Dermatol Venereol 1: S3-8. doi: 10.1016/S0151 $9638(10) 70002-8$

Parkes J, Guha IN, Harris S, Rosenberg WM, Roderick PJ (2012) Systematic review of the diagnostic performance of serum markers of liver fibrosis in alcoholic liver disease. Comp Hepatol 11: 5. doi: 10.1186/1476-5926-11-5

Peters L, Mocroft A, Soriano V, Resino S, Bellón JM, Asensio C, Rockstroh J, Rauch A, Karlsson A, Knysz B, Pradier C, Zilmer K Lundgren JD, for EuroSIDA in EuroCoord (2013) Hyaluronic acid levels predict risk of hepatic encephalopathy and liver-related death in HIV/viral hepatitis coinfected patients. PLoS One 8: e64283. doi: 10.1371/journal.pone.0064283

Resino S, Bellón JM, Asensio C, Micheloud D, Miralles P, Vargas A, Catalán P, López JC, Alvarez E, Cosin J, Lorente R, MuñozFernández MA, Berenguer J (2010) Can serum hyaluronic acid replace simple non-invasive indexes to predict liver fibrosis in HIV/Hepatitis C coinfected patients? BMC Infect Dis 10: 244. doi: 10.1186/1471-2334-10-244

Rossi E, Adams LA, Bulsara M, Jeffrey GP (2007) Assessing liver fibrosis with serum marker models. Clin Biochem Rev 28: 3-10

Rostami S, Parsian H (2013) Hyaluronic Acid: from biochemical characteristics to its clinical translation in assessment of liver fibrosis. Hepat Mon 14: e13787. doi: 10.5812/hepatmon.13787

Salmon-Ceron D, Lewden C, Morlat P, Bévilacqua S, Jougla E, Bonnet F, Héripret L, Costagliola D, May T, Chêne G, Mortality 2000 study group (2005) Liver disease as a major cause of death among HIV infected patients: role of hepatitis $\mathrm{C}$ and $\mathrm{B}$ viruses and alcohol. J Hepatol 42: 799-805

Schanté C, Zuber G, Herlin C, Vandamme TF (2011) Chemical modifications of hyaluronic acid for the synthesis of derivatives for a broad range of biomedical applications. Carbohydrate Polymers 85: 469-489

Schilsky ML. Hepatitis B “360” (2013) Transplantation Proceedings 45: 982-985

Seven G, Karatayli SC, Köse SK, Yakut M, Kabaçam G, Törüner M, Heper AO, Voelker M, Erden E, Bozdayi AM, Uzunalımoğlu O, Bozkaya H, Yurdaydin C (2011) Serum connective tissue markers as predictors of advanced fibrosis in patients with chronic hepatitis B and D. Turk J Gastroenterol 22: 305-314

Shen Y, Shi G, Huang C, Zhu X, Chen S, Sun H, Zhou J, Fan J (2015) Prediction of post-operative liver dysfunction by serum markers of liver fibrosis in hepatocellular carcinoma. PLoS One 10: e0140932. doi: 10.1371 /journal.pone.0140932

Sowa JP, Heider D, Bechmann LP, Gerken G, Hoffmann D, Canbay A (2013) Novel algorithm for non-invasive assessment of fibrosis in NAFLD. PLoS One 8: e62439. doi: 10.1371/journal.pone.0062439 Stachowska E, Maciejewska D, Ossowski P, Drozd A, Ryterska K, Banaszczak M, Milkiewicz M, Raszeja-Wyszomirska J, Slebioda M, Milkiewicz P, Jelen H (2013) Apolipoprotein E4 allele is associated with substantial changes in the plasma lipids and hyaluronic acid content in patients with nonalcoholic fatty liver disease. J Physiol Pharmacol 64: 711-717.

Stickel F, Poeschl G, Schuppan D, Conradt C, Strenge-Hesse A, Fuchs FS, Hofmann WJ, Seitz HK (2003) Serum hyaluronate correlates with histological progression in alcoholic liver disease. Eur I Gastroenterol Hepatol 15: 945-950

Valva P, Casciato P, Diaz Carrasco JM, Gadano A, Galdame O, Galoppo MC, Mullen E, De Matteo E, Preciado MV (2011) The role of serum biomarkers in predicting fibrosis progression in pediatric and adult hepatitis $\mathrm{C}$ virus chronic infection. PLoS One 6: e23218. doi: 10.1371 /journal.pone.0023218

Voumvouraki A, Koulentaki M, Notas G, Sfakianaki O, Kouroumalis E (2011) Serum surrogate markers of liver fibrosis in primary biliary cirrhosis. Eur J Intern Med 22: 77-83. doi: 10.1016/j.ejim.2010.10.002

Yu X, Deng M, Zhang K, Xie XS (2015) Clinical Analysis of 36 Elderly Patients with Schistosome-Induced Liver Disease Complicated by Hepatitis E. Zhongguo Ji Sheng Chong Xue Yu Ji Sheng Chong Bing ZaZhi 33: 258-263 